\title{
Effects of sit-to-stand imagery group training on balance performance in individuals with chronic hemiparetic stroke: a randomized control trial
}

\author{
Jeongwon Lee ${ }^{\mathrm{a}}$, Sujin Hwang ${ }^{\mathrm{b}}$, Sinae Ahn ${ }^{\mathrm{a}}$ \\ aDepartment of Occupational Therapy, Yeoju Institute of Technology, Yeoju, Republic of Korea \\ ${ }^{\mathrm{b}}$ Department of Physical Therapy, Division of Health Science, Baekseok University, Cheonan, Republic of Korea
}

Objective: To evaluate the effects of sit-to-stand (STS) imagery group training (IGT) on STS movement and balance performance for chronic hemiparetic stroke.

Design: Randomized controlled trial.

Methods: A total of 30 people with chronic hemiparetic stroke (15 for STS-IGT group and 15 for control) were recruited in this study. The STS-IGT group participated in a videotape-based STS-IGT for 30 minutes a day, five days a week for six weeks, while the control group watched a documentary on television for the same period. The STS-IGT focused on a five-stage protocol. Specifically, external imagery was used during the four phases of the STS movement from the seat of the chair to standing up. All of the participants also participated in a regular rehabilitation program. STS movement and balance performance were assessed using three clinical measures.

Results: After training, time to perform the 5-repetition STS test significantly increased in the STS-IGT group (change value, $4.0 \pm 2.0 \mathrm{sec}$ ) compared with the control group (change value, $0.9 \pm 0.7 \mathrm{sec})(p<0.05)$. There was a greater improvement in Berg balance scale scores in the STS-IGT group (change value, $0.2 \pm 4.1$ points) compared with the control group (change value, $0.3 \pm 0.9$ points) $(p<0.05)$. There was a greater improvement in Timed Up and Go scores in the STS-IGT group (change value, $2.6 \pm 1.5 \mathrm{sec})$ compared with the control group (change value, $0.9 \pm 1.0 \mathrm{sec})(p<0.05)$.

Conclusions: STS-IGT can be considered as a useful option for restoration of STS movement and balance performance for individuals with chronic hemiparetic stroke who are unable to fully participate in physical activities.

Key Words: Imagery, Standing, Stroke

\section{Introduction}

Sit-to-stand (STS) movement is one of the prerequisite abilities for walking, daily living, and engaging within the community because it is used for making positional changes from a sitting to standing position that are required to perform functional activities. Dysfunctional STS movement would lead to impaired performance of functional activities of daily living (ADL) and community [1].

One of the major problems that may cause a dysfunctional
STS movement is muscle weakness of the lower extremity. Stroke survivors encounter motor deficiencies, such as coordination deficits, spasticity, lack of endurance, and impaired postural control. However, weakness of the paretic limb is the most commonly reported problem for upright standing or STS movement [2]. Previous studies have reported that individuals with hemiparetic stroke demonstrate an increase in STS movement time when compared to older adults without neurological impairments [3-5]. Trunk instability often occurs following a stroke and may lead to asymmetrical

Received: 30 March, 2016 Revised: 15 June, 2016 Accepted: 15 June, 2016

Corresponding author: Sinae Ahn

Department of Occuaptional Therapy, Yeoju Institute of Technology, 338 Sejong-ro, Yeoju 12652, Republic of Korea

Tel: 82-31-880-5393 Fax: 82-31-886-8925 E-mail: otlovesn@gmail.com

(c) This is an Open-Access article distributed under the terms of the Creative Commons Attribution Non-Commercial License (http://creativecommons.org/licens es/by-nc/4.0) which permits unrestricted non-commercial use, distribution, and reproduction in any medium, provided the original work is properly cited.

Copyright @ 2016 Korean Academy of Physical Therapy Rehabilitation Science 
weight shiftings in both lower extremities during functional tasks, such as the STS movement [6].

Individuals with hemiparetic stroke automatically use their paretic limb less during functional activities, such as upright stance. Generally, hemiparetic stroke patients do not perceive their weight-shifting performance accurately during the STS movement. Most physical training interventions rely on this sense to improve symmetry during the execution of various functional tasks. Therefore, having a perception of motor performance is essential to improve functional activities for individual with hemiparetic stroke. The sensory information would help improve clinical interventions aimed at reducing asymmetries during STS movement by indicating which aspect should be emphasized in the instructions given to the patients [7].

Motor imagery training is a useful alternative approach for physical rehabilitation following stroke, and offers a safe, available, and inexpensive treatment method that is the use at home without specific equipment. Motor imagery training is independent from physical performance of an impaired limb, and allows for use of the brain to in reestablishing the circuitry that mediates voluntary movement. The training can create cortical plasticity changes similar to those created following physical activity, thus, these mechanisms point to the potential of using motor imagery practice in the neurological rehabilitation of individuals following stroke [8].

Malouin et al. [9] found that after just one session of motor imagery training in an STS task, 12 stroke patients demonstrated an enhancement of weight bearing on the paretic lower limb while standing, with the improvement maintained after 24 hours. Oh et al. [10] reported that motor imagery training has a positive effect on the symmetrical use of knee extensors during STS and stand-to-sit tasks. Guttman et al. [11] suggested that the imagery practice of meaningful motor tasks can positively affect real performance, including STS and reaching to grasp objects. Recently, some researchers reported the effects of motor imagery group therapy on upper extremity activities as well as gait performance to individuals with stroke [12-14]. Page et al. [13] demonstrated that physical upper extremity ADL practice combined with audiotape group therapy for mental practice resulted in greater therapeutic benefits to the experimental group than those achieved by physical practice alone. Riccio et al. [14] demonstrated the advantage of adding group mental practice to physical practice in treatment of the affected upper extremity in post-stroke patients. Dickstein et al. [12] examined whether the application of a group-based motor imagery practice to community-dwelling individuals with chronic hemiparesis improved gait. They reported that the verbal reports of most participants alluded to satisfaction with the intervention and to an increase in self-confidence, although comparisons within (pre vs. post) and between treatments (experimental vs. control) indicated no significant change in any gait variables. Therefore, there is the lack of evidence for the effectiveness of group-based motor imagery training in improving upper and lower extremities activities among individuals following hemiparetic stroke.

The purpose of this study was to investigate the effects of STS imagery group training (IGT) on STS movement and balance performance in individuals with chronic hemiparetic stroke. The hypothesis of this study was that the STS-IGT would produce greater improvement on STS movement and balance performance than conventional training in individuals with chronic hemiparetic stroke.

\section{Methods}

\section{Subjects}

Voluntary participants were recruited using a brochure describing the study purpose, inclusion and exclusion criteria, treatment period, and information about the researchers from a local rehabilitation center. All participants signed an informed consent form after they received information about the study's purpose, study's method, possible benefits and risks, privacy, and use of data. Thirty participants with chronic hemiparetic stroke were recruited and were classified into either the STS-IGT group (15 persons) or the control group (15 persons). The inclusion criteria were (1) patients with six months or more since first stroke onset, (2) ability to walk independently over ground for at least $10 \mathrm{~m}$ with or without use of an ankle foot orthosis or assistive device, (3) absence of any cognitive impairment (scores above 24 on the MiniMental State Examination) [15], (4) ability to understand verbal instructions, (5) no cerebellar lesions, (6) no significant body or visuospatial hemi-neglect, (7) a good ability for imagery functioning (a score of 32 or higher on the revision of Movement Imagery Questionnaire) [16], (8) absence of neurological disorders except for stroke and (9) absence of orthopedic conditions. The participant was excluded if he/she reported serious visual or somatosensory impairments and post bilateral stroke. Table 1 shows the general characteristics of the STS-IGT and control groups. 


\section{Procedures}

This study was a pretest-posttest control group design with a six-week intervention. Outcome measures were taken the day before and after the six-week training. During the treatment period, the STS-IGT group was involved in STS-IGT for 30 minutes a day, five days a week for six weeks, while the control group watched documentary programs on television (TV) for same period. All participants received conventional rehabilitation program.

The participants in the STS-IGT group participated in six weeks of STS-IGT, 30 minutes a day, five times a week for six-weeks, while participants in the control group watched documentary programs on TV on topics related to health for the same period of time (Figure 1).

Two different videotapes (normal young adults male vs. each patient) were used for STS-IGT. One videotape showed a normal young adult male from anterior, left and right views while performing the STS activity on a back-chair or stool. The first videotape showed the man performing an STS

Table 1. Common characteristics of the participants $\quad(\mathrm{N}=30)$

\begin{tabular}{lcc}
\hline \multirow{2}{*}{ Variable } & \multicolumn{2}{c}{ Participants } \\
\cline { 2 - 3 } & $\begin{array}{c}\text { STS-IGT group } \\
(\mathrm{n}=15)\end{array}$ & $\begin{array}{c}\text { Control group } \\
(\mathrm{n}=15)\end{array}$ \\
\hline Age (y) & $53.9(9.59)$ & $57.2(14.57)$ \\
Sex (male/female) & $9 / 6$ & $6 / 9$ \\
Height $(\mathrm{cm})$ & $161.6(10.6)$ & $160.3(12.2)$ \\
Weight $(\mathrm{kg})$ & $62.7(10.7)$ & $61.3(12.6)$ \\
Etiology (ischemic/hemorrhage) & $11 / 4$ & $9 / 6$ \\
Brunnstrom stage $(3 / 4 / 5 / 6)$ & $2 / 5 / 6 / 2$ & $5 / 6 / 4 / 0$ \\
\hline
\end{tabular}

Values are presented as $\mathrm{n}(\%)$ or mean (SD). STS-IGT: sit-to-stand imagery group training. movement at a slow, comfortable speed, and the rest of the tape showed the man perform the STS at a normal speed. The other videotape showed each patient from anterior, left and right views while he/she performed the STS movement at a comfortable speed. Filming was carried out twice, before training and three weeks after training, and was used to show each patient's information about their performance of their STS movement in training program.

The first week of the six-week training focused on familiarizing patients with normal STS movement sequences and identifying their own problems with the STS movement by studying the differences between the participant's STS movement before training from a normal young adult male's STS movement. The therapist explained the kinematic changes in each phase of the buttocks, ankle joints, hip joints, and trunk position. The therapist explained the kinematic changes of the buttocks, ankle joints, hip joints and trunk position during the four phases of the STS movement.

During the last five weeks, the STS-IGT group performed IGT according to a five-stage protocol.

\section{Protocol}

To treat STS-IGT in individuals with chronic hemiparetic stroke, this study used five stage protocols: progressive relaxation, external imagery (analysis of STS sequences), problem identification (each patient's videotape), internal imagery (first-person perspective), and mental rehearsal. This protocol was modified based on the Hwang et al.'s locomotor imagery training [17]. A detail description of the five-stage protocol is shown as follows:

(1) The progressive relaxation stage aims to increase blood flow to the brain by decreasing the respiratory rate, heart rate, blood pressure and muscle tension. The stage uses the Benson's relaxation protocol. Then the patients were

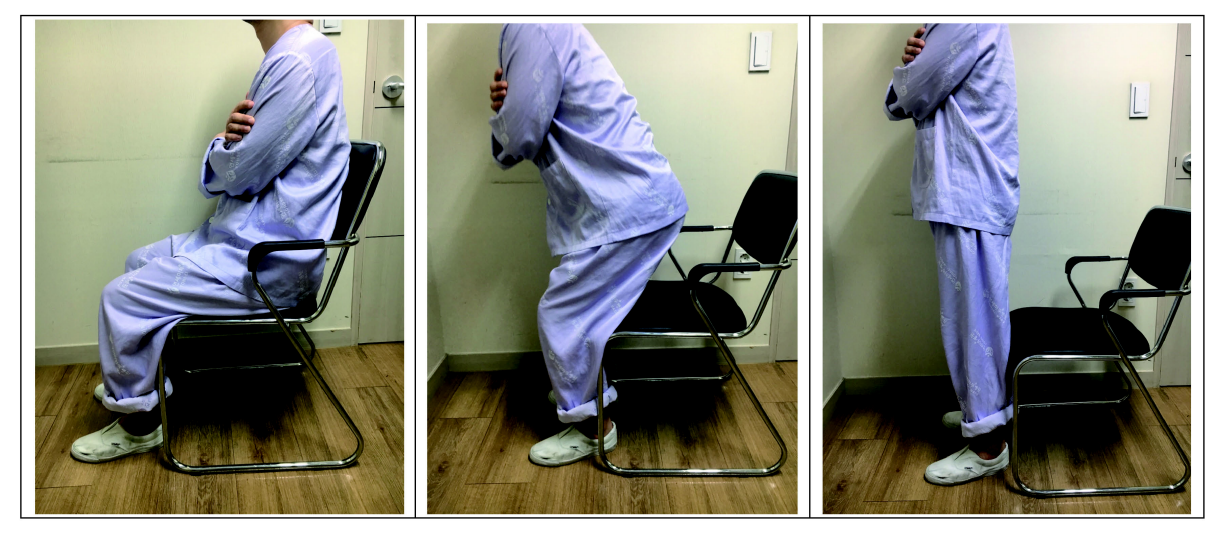

Figure 1. The view of a participant in this study for sit-to-stand. 
asked to concentrate and to be aware of their own breathing while they repeated the breathing pattern.

(2) The external imagery stage aims to analyze the STS sequences. In this stage, the participants try to analyze the STS sequences in order to understand the STS movement and to facilitate motor planning and problem identification. The therapist shows the videotape of the normal young adult's STS movement. The patient closely views the videotape of a normal, slow, comfortable STS movement as if he/she is watching a movie.

(3) The problem identification stage is used to understand the participant's own STS movement dysfunction and to compare his/her STS movement with that of a normal young adult.

(4) The internal imagery stage involves the subject imagining walking in first-person perspective using the normal young adult's STS movement. The videotape in this stage is the same as the videotape used for the external imagery stage.

(5) Finally, the intention of the mental rehearsal stage is for the patients to imagine normal STS movement. The participant is asked to mentally replay from the external imagery stage to the problem identification stage of the five-stage protocol, closing their eyes for two minutes after each STS movement imagery stage. After the mental rehearsal stage, the participant is asked to verbalize one difference between the normal STS movement sequences and their own STS movement. There is also an identifying period when the participant performed motor imagery.

The STS imagery training in this study focused on four phases of the STS movement used in the external imagery stage: Phase I starts with initiation of the movement and ends just before the buttocks are lifted from the seat of the chair. Phase II begins as the buttocks are lifted and ends when maximal ankle dorsiflexion is achieved. Phase III is initiated just after maximum ankle dorsiflexion and ends when the hips first cease to extend, including lower extremity and trunk extension. Phase IV begins after hip extension is reached and ends when all motion associated with stabilization is completed [1].

\section{Outcome measures}

\section{5-repetition sit-to-stand test}

The 5-repetition STS test has been introduced as an outcome measure in studies investigating strength training and functional performance in subjects with chronic stroke, as well as cross-sectional studies evaluating the association of disabilities and falls in population with stroke [18] (Figure 1). The 5-repetition STS test measured the time taken to complete 5 repetitions of the STS maneuver. Individuals were seated on an armless, height-controlled chair, which was adjusted to allow for approximately 90-degree angles at the hip and knee joints. The distance between their feet was not constrained and the STS task was performed with shoes on. Individuals performed three self-paced, followed by three fast-paced STS trials without the use of their arms. The first 3 trials were for familiarization purposes, and the average of the next 3 trials was used for analysis. A one-minute rest period was given between trials to prevent fatigue. Standardized instructions were given as follows: "by the count of 3, please stand up and sit down as quickly as possible for 5 times. Place your hands on your lap and do not use them throughout the procedure. Lean your back against the chair's backrest at the end of every repetition." The timing started once the participant's back left the backrest and stopped once the back touched the backrest. The STS test has an excellent intrarater reliability of intraclass correlation coefficient (ICC) (range, 0.97-0.98), interrater reliability ( $\mathrm{ICC}=1.00)$, and test-retest reliability (ICC range, 0.99-1.00) in individuals with chronic hemiparetic stroke $[18,19]$.

\section{Berg balance scale}

The Berg balance scale (BBS) was used to assess the participant's ability to maintain stability. The BBS is a widely used test for the assessment of postural control while sitting, standing, and transferring in elderly individuals and individuals with neurological disorders. The BBS uses a five-point ordinal scale ranging from 0 (disability) to 4 (complete independent performance) and consists of 14 items; the maximum possible score is 56 points. The scale has been shown to be reliable and valid in stroke patients. A cut-off score of 45 points is used for fall prediction. The BBS involves 14 tasks; each has a score between 0 and 4, adding up to a total score of 56. An excellent reliability (ICC range, 0.98-0.99) was found in individuals with chronic stroke for the BBS [20-22].

\section{Timed Up and Go test}

The Timed Up and Go (TUG) test was designed for the evaluation of balance and basic functional mobility to determine the risk of falls [23]. In this study, a participant sat with his/her back against a chair with an armrest. On the cue 'Go', the patient was asked to stand up, walk at a comfortable speed to a marked point, which was $3 \mathrm{~m}$ from the chair, 
turn around, walk back, and sit down in the chair. All participants wore comfortable, regular footwear and used their customary walking aid during the test. The time taken to complete the task was measured using a stopwatch. The test was performed three times, and the average value was recorded. The intrarater $(\mathrm{r}=0.99)$ and inter-rater $(\mathrm{r}=0.98)$ reliability was high $[23,24]$.

\section{Statistical analysis}

The independent variable in this study was the STS-IGT. The dependent variables were the 5-repetition STS test, BBS, and TUG test. General characteristics of the participants were analyzed using descriptive statistics. Independent $t$-tests were used to determine whether the changes from the pretest to the posttest of the clinical parameters differed significantly between the STS-IGT and control groups. An alpha level of $p<0.05$ was used as statistical significance. All statistical analyses were performed using the (SPSS Inc., Chicago, IL, USA).

\section{Results}

Descriptive statistics and independent t-test values of both groups of all dependent variables are presented in Tables 2 and 3 , respectively. Table 2 shows the pre-test data of the three outcome measures for both groups. The data of the three outcome measures were not significantly different in STS-IGT group compared to control group. Table 3 also shows the pre-test and post-test data of the three outcome measures for both groups after analyzing the descriptive statistics and independent t-test. After training, the 5-repetition STS test had significantly increased in the STS-IGT group (change time, $4.0 \pm 2.0 \mathrm{sec}$ ) more than the control group (change time, $0.9 \pm 0.7 \mathrm{sec}$ ). There was a greater improvement in BBS scores in the STS-IGT group (change value, 7.2 \pm 4.1 points) compared with the control group (change value, $0.3 \pm 0.9$ points). The TUG test was significantly improved in the STS-IGT group (change time, $2.6 \pm 1.5 \mathrm{sec}$ ) compared with the control group (change time, $0.9 \pm 1.0 \mathrm{sec}$ ).

\section{Discussion}

This study examined the effects of STS-IGT for improving the STS movement and balance performance frequently used in everyday activities, which are is commonly dysfunctional in individuals with chronic hemiparetic stroke. After training, the findings of this study were as follows: first, the 5-repetition STS test significantly increased in the STS-IGT group compared with the control. Second, there were greater improve ent of BBS scores in the STS-IGT group compared with the control group. Finally, TUG scores were lower in the STS-IGT group compared with the control group. Therefore, STS-IGT is effective on the STS movement and balance performance, and the results of this study has well established the evidence for the effectiveness of IGT in individuals with chronic hemiparetic stroke.

The STS movement includes movement of the center of mass of the body from a sit to standing position without los-

Table 2. Comparison of pre-test data between STS-IGT and control groups

$(\mathrm{N}=30)$

\begin{tabular}{ccccc}
\hline Variable & STS-IGT group (n=15) & Control group (n=15) & t & $p$ \\
\hline Timed Up and Go test (sec) & $15.1(3.5)$ & $14.8(3.0)$ & -0.529 & 0.601 \\
Berg balance scale (points) & $45.9(4.3)$ & $46.0(5.6)$ & 0.073 & 0.942 \\
5-repetition STS test (sec) & $17.0(2.2)$ & $18.3(2.8)$ & 1.391 & 0.175 \\
\hline
\end{tabular}

Values are presented as mean (SD).

STS-IGT: sit-to-stand imagery group training.

Table 3. Comparisons of treatment effects between STS-IGT and control groups

$(\mathrm{N}=30)$

\begin{tabular}{|c|c|c|c|c|c|c|}
\hline \multirow{2}{*}{ Variable } & \multicolumn{2}{|c|}{ STS-IGT group $(n=15)$} & \multicolumn{2}{|c|}{ Control group $(\mathrm{n}=15)$} & \multirow{2}{*}{$\mathrm{t}$} & \multirow{2}{*}{$P$} \\
\hline & Pre-test & Post-test & Pre-test & Post-test & & \\
\hline Timed Up and Go test (sec) & $15.1(3.5)$ & $12.5(2.9)$ & $14.5(3.0)$ & $13.6(2.7)$ & -3.879 & 0.001 \\
\hline Berg balance scale (points) & $45.9(4.3)$ & $53.1(2.2)$ & $46.0(5.6)$ & $46.3(5.4)$ & -6.400 & $<0.001$ \\
\hline 5-repetition STS test $(\mathrm{sec})$ & $17.0(2.2)$ & $13.0(1.6)$ & $18.3(2.81)$ & $17.4(2.5)$ & -5.547 & $<0.001$ \\
\hline
\end{tabular}

Values are presented as mean (SD).

STS-IGT: sit-to-stand imagery group training. 
ing balance, as well as maintaining balance from the sitting position to an erect stance. In individuals with chronic hemiparetic stroke, the STS movement is affected by lower extremity muscle weakness and balance dysfunction. Previous studies expected to reduce the duration of STS movement and to improve the symmetry between both lower extremities on the rise phase and final stance after STS movement training [6,7,25]. Lomaglio and Eng [25] reported that paretic muscle strength and the ability to load the paretic limb are important factors underlying the ability to rise from a chair in individuals with chronic stroke. Recently, motor imagery of STS movement for individuals with stroke has been introduced by some researchers $[10,11]$. Previous studies have reported that the application of motor imagery practice can be an effective means of enhancing STS movement for treatment of hemiplegic stroke patients. The results of this study also suggested the reduction of time on STS movement after STS-IGT. The time to perform the 5-repetition STS test as well as the TUG was significantly decreased after the STS-IGT.

Brière et al. [7] suggested that individuals with poststroke had a higher perception of their weight-bearing distribution than their actual ability to distribute weight at the knees, and try to produce higher effort with their paretic knee while standing up. They also reported that impaired perception interferes or limits rehabilitation processing in individuals with post-stroke. STS-IGT for persons with hemiparetic stroke is a group-based motor imagery approach that improves STS movement without requiring actual movements by the lower limbs. Therefore, STS-IGT may be beneficial for patients who are unable to participate in physical STS training secondary to fatigue, severe paralysis or impaired balance as well as perceptual impairment.

The postural parameters could assist to identify asymmetric dynamic and static posture, which has been indicated as one of the prevalent locomotor deficits of stroke-related hemiparesis [26,27]. Although this study did not measure the biomechanical factors, such as kinematics and kinetics, the results of this study could not directly identify the asymmetric posture of the participants. Our results showed increased BBS scores post STS-IGT which may suggest a positive improvement in static and dynamic balance.

Motor imagery training cannot identify immediately whether the imagery performance is correct or incorrect because the training does not involve any actual movement during the training period. This study used the problem identification stage in the five-stage protocol to correct the in- correct imagery performance during training. The stage is used to understand the participant's own STS movement dysfunction and to compare his/her STS movement with that of a normal young adult. This stage is derived from the augmented feedback concept of motor learning theory to provide perceptual feedback information about the participant's own STS movement pattern.

This study used the group training methods for STS imagery training in individuals with chronic hemiparetic stroke. It is often necessary to relearn functional activities via group-centered therapeutic approaches due to issues of accessibility, cost, and human resources in rehabilitation surroundings for stroke patients [12,28-32]. Tamir et al. [31] reported that the combination of motor imagery and real practice in group treatment may be effective in patients with Parkinson's disease, and allows for the extension of practice time with negligible risk and low cost. Long et al. [32] suggested that imagery rescripting and exposure group treatment can be adapted to successfully reduce distress associated with combat Veterans' chronic posttraumatic nightmares. Although those studies did not involve patients with chronic stroke, the results of this study agree with our results, showing the positive effect of IGT.

It is possible that IGT would produce a positive effect on STS movement and balance performance. Dickstein et al. [12] suggested that the positive reinforcement induced by motor imagery gait practice may explain the subject's motivation and courage developed to practice gait activities required for daily living for chronic stroke patients. However, this study did not measure the patients' satisfaction levels and did not add any physical training in our protocol. Future studies will need to consider the relationship between motor imagery and physical activities. Also, this study did not measure any biomechanical factors, such as kinematics and kinetics, to examine the treatment effects. Further studies should include assessment of other parameters, such as patient's satisfaction levels and biomechanical factors.

\section{Conflict of Interest}

The authors declared no potential conflicts of interest with respect to the authorship and/or publication of this article.

\section{References}

1. Janssen WG, Bussmann HB, Stam HJ. Determinants of the 
sit-to-stand movement: a review. Phys Ther 2002;82:866-79.

2. Kim CM, Eng JJ. The relationship of lower-extremity muscle torque to locomotor performance in people with stroke. Phys Ther 2003;83:49-57.

3. Silva A, Sousa AS, Pinheiro R, Ferraz J, Tavares JM, Santos R, et al. Activation timing of soleus and tibialis anterior muscles during sit-to-stand and stand-to-sit in post-stroke vs. healthy subjects. Somatosens Mot Res 2013;30:48-55.

4. Prudente C, Rodrigues-de-Paula F, Faria CD. Lower limb muscle activation during the sit-to-stand task in subjects who have had a stroke. Am J Phys Med Rehabil 2013;92:666-75.

5. Boyne P, Israel S, Dunning K. Speed-dependent body weight supported sit-to-stand training in chronic stroke: a case series. J Neurol Phys Ther 2011;35:178-84.

6. Mazzà C, Stanhope SJ, Taviani A, Cappozzo A. Biomechanic modeling of sit-to-stand to upright posture for mobility assessment of persons with chronic stroke. Arch Phys Med Rehabil 2006;87:635-41.

7. Brière A, Nadeau S, Lauzière S, Gravel D. Perception of weightbearing and effort distribution during sit-to-stand in individuals post-stroke. Percept Mot Skills 2013;117:1208-23.

8. Sun L, Yin D, Zhu Y, Fan M, Zang L, Wu Y, et al. Cortical reorganization after motor imagery training in chronic stroke patients with severe motor impairment: a longitudinal fMRI study. Neuroradiology 2013;55:913-25.

9. Malouin F, Belleville S, Richards CL, Desrosiers J, Doyon J. Working memory and mental practice outcomes after stroke. Arch Phys Med Rehabil 2004;85:177-83.

10. Oh DW, Kim JS, Kim SY, Yoo EY, Jeon HS. Effect of motor imagery training on symmetrical use of knee extensors during sit-to-stand and stand-to-sit tasks in post-stroke hemiparesis. NeuroRehabilitation 2010;26:307-15.

11. Guttman A, Burstin A, Brown R, Bril S, Dickstein R. Motor imagery practice for improving sit to stand and reaching to grasp in individuals with poststroke hemiparesis. Top Stroke Rehabil 2012;19:306-19.

12. Dickstein R, Levy S, Shefi S, Holtzman S, Peleg S, Vatine JJ. Motor imagery group practice for gait rehabilitation in individuals with post-stroke hemiparesis: a pilot study. NeuroRehabilitation 2014;34:267-76.

13. Page SJ, Levine P, Leonard A. Mental practice in chronic stroke: results of a randomized, placebo-controlled trial. Stroke 2007; 38:1293-7.

14. Riccio I, Iolascon G, Barillari MR, Gimigliano R, Gimigliano F. Mental practice is effective in upper limb recovery after stroke: a randomized single-blind cross-over study. Eur J Phys Rehabil Med 2010;46:19-25.

15. Folstein MF, Folstein SE, McHugh PR. "Mini-mental state". A practical method for grading the cognitive state of patients for the clinician. J Psychiatr Res 1975;12:189-98.

16. Sharma N, Pomeroy VM, Baron JC. Motor imagery: a backdoor to the motor system after stroke? Stroke 2006;37:1941-52.
17. Hwang S, Jeon HS, Yi CH, Kwon OY, Cho SH, You SH. Locomotor imagery training improves gait performance in people with chronic hemiparetic stroke: a controlled clinical trial. Clin Rehabil 2010;24:514-22.

18. Mong Y, Teo TW, Ng SS. 5-repetition sit-to-stand test in subjects with chronic stroke: reliability and validity. Arch Phys Med Rehabil 2010;91:407-13.

19. Bohannon RW. Test-retest reliability of the five-repetition sit-to-stand test: a systematic review of the literature involving adults. J Strength Cond Res 2011;25:3205-7.

20. Blum L, Korner-Bitensky N. Usefulness of the berg balance scale in stroke rehabilitation: a systematic review. Phys Ther 2008;88:559-66.

21. Bogle Thorbahn LD, Newton RA. Use of the berg balance test to predict falls in elderly persons. Phys Ther 1996;76:576-83; discussion 584-5.

22. Berg K, Wood-Dauphinee S, Williams JI. The Balance Scale: reliability assessment with elderly residents and patients with an acute stroke. Scand J Rehabil Med 1995;27:27-36.

23. Podsiadlo D, Richardson S. The timed "Up \& Go": a test of basic functional mobility for frail elderly persons. J Am Geriatr Soc 1991;39:142-8.

24. Ng SS, Hui-Chan CW. The timed up \& go test: its reliability and association with lower-limb impairments and locomotor capacities in people with chronic stroke. Arch Phys Med Rehabil 2005;86:1641-7.

25. Lomaglio MJ, Eng JJ. Muscle strength and weight-bearing symmetry relate to sit-to-stand performance in individuals with stroke. Gait Posture 2005;22:126-31.

26. Winstein CJ, Gardner ER, McNeal DR, Barto PS, Nicholson DE. Standing balance training: effect on balance and locomotion in hemiparetic adults. Arch Phys Med Rehabil 1989;70:755-62.

27. Nichols DS. Balance retraining after stroke using force platform biofeedback. Phys Ther 1997;77:553-8.

28. Leung DP, $\mathrm{Ng} \mathrm{AK}$, Fong KN. Effect of small group treatment of the modified constraint induced movement therapy for clients with chronic stroke in a community setting. Hum Mov Sci 2009;28:798-808.

29. Brogårdh C, Sjölund BH. Constraint-induced movement therapy in patients with stroke: a pilot study on effects of small group training and of extended mitt use. Clin Rehabil 2006;20:218-27.

30. Dean C. Group task-specific circuit training for patients discharged home after stroke may be as effective as individualised physiotherapy in improving mobility. J Physiother 2012;58:269.

31. Tamir R, Dickstein R, Huberman M. Integration of motor imagery and physical practice in group treatment applied to subjects with Parkinson's disease. Neurorehabil Neural Repair 2007;21:68-75.

32. Long ME, Hammons ME, Davis JL, Frueh BC, Khan MM, Elhai $\mathrm{JD}$, et al. Imagery rescripting and exposure group treatment of posttraumatic nightmares in Veterans with PTSD. J Anxiety Disord 2011;25:531-5. 